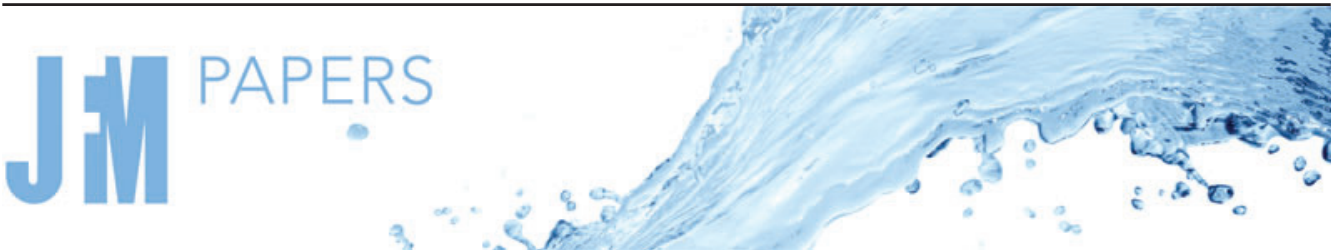

\section{Formation of spiral waves in cylindrical containers under orbital excitation}

\author{
G.M. Horstmann ${ }^{1,2, \dagger, ~ S . ~ A n d e r s ~}{ }^{1}$, D.H. Kelley ${ }^{2}$ and T. Weier ${ }^{1}$ \\ ${ }^{1}$ Institute of Fluid Dynamics, Helmholtz-Zentrum Dresden-Rossendorf, Bautzner Landstrasse 400, \\ 01328 Dresden, Germany \\ ${ }^{2}$ Department of Mechanical Engineering, University of Rochester, Rochester, NY 14627, USA
}

(Received 6 January 2021; revised 5 July 2021; accepted 28 July 2021)

The lowest swirling wave mode arising in upright circular cylinders as a response to circular orbital excitation has been widely studied in the last decade, largely due to its high practical relevance for orbitally shaken bioreactors. Our recent theoretical study (Horstmann et al., J. Fluid Mech., vol. 891, 2020, A22) revealed a damping-induced symmetry breaking mechanism that can cause spiral wave structures manifested in the so far widely disregarded higher rotating wave modes. Building on this work, we develop a linear criterion describing the degree of spiralisation and classify different spiral regimes as a function of the most relevant dimensionless groups. The analysis suggests that high Bond numbers and shallow liquid layers favour the formation of coherent spiral waves. This result paved the way to find the predicted wave structures in our interfacial sloshing experiment. We present two sets of experiments, with different characteristic damping rates, verifying the formation of both coherent and overdamped spiral waves in conformity with the theoretical predictions.

Key words: wave-structure interactions

\section{Introduction}

Ranging from galaxy-scale accretion discs (Boffin 2001) via atmospheric cyclones (Nolan \& Zhang 2017) down to the human heart (Gray \& Jalife 1996), rotating spiral waves are a common phenomenon in nature, which may occur in very diverse and physically different environments. Besides apparent topological similarities such as that all spirals possess a pronounced chirality, it is interesting to note that all the known kinds of spiral waves, most prominent in excitable media and reaction-diffusion systems, are inherently

$†$ Email address for correspondence: g.horstmann@hzdr.de

(C) The Author(s), 2021. Published by Cambridge University Press. This is an Open Access article, distributed under the terms of the Creative Commons Attribution licence (http://creativecommons.org/ licenses/by/4.0/), which permits unrestricted re-use, distribution, and reproduction in any medium, provided the original work is properly cited. 


\section{G.M. Horstmann, S. Anders, D.H. Kelley and T. Weier}

nonlinear phenomena. Therefore, it came as a slight surprise to us that our recently published formulation of interfacial waves in upright circular cylinders (Horstmann, Herreman \& Weier 2020) predicted the formation of non-propagating spiral wave patterns under the influence of viscous damping as a linear response to orbital excitation.

The interest in liquid sloshing dynamics of partially filled containers arose in the 1950 s, in those days mainly with the focus on spacecraft and naval applications. Since then, sloshing dynamics has been investigated in a wide variety of geometries as a response to a multitude of excitation modes (Faltinsen \& Timokha 2009). Yet, the specific case of orbitally shaken cylinders, i.e. cylindrical containers following a perfect circular trajectory, has gained attention only recently, basically in light of three different fields of interest. First, orbitally shaken bioreactors (Klöckner \& Büchs 2012) were identified as an important application in the last decade, where gas transfer, mixing dynamics and shear stresses are essentially imposed by the wave motion. To this end, wave and flow dynamics has been studied in several experiments with the aim to better assess the operating parameters (see e.g. Reclari et al. 2014; Weheliye et al. 2018; Alpresa et al. 2018). Second, it is of fundamental interest to understand the mean mass transport induced by swirling waves, an old problem that dates back to Prandtl (1949). Significant progress on this issue has recently been made by Bouvard, Herreman \& Moisy (2017) and Faltinsen \& Timokha (2019). And third, the hydrodynamic similarity to the magnetohydrodynamic 'metal pad roll instability', a potential limiting factor in aluminium reduction cells and liquid-metal batteries (Weber et al. 2017; Horstmann, Weber \& Weier 2018; Herreman et al. 2019; Politis \& Priede 2021), was utilised by Horstmann, Wylega \& Weier (2019). We introduced a multilayer orbital sloshing experiment, allowing us to imitate the wave motion as it can arise from the metal pad roll instability.

In two- and three-layer stratifications, the interfacial wave motion is subject to considerably stronger damping forces as compared to free-surface waves, rendering it impossible to apply existing inviscid sloshing models. We addressed this issue recently in Horstmann et al. (2020), where we presented a hybrid model on orbital sloshing of both free-surface and two-layer interfacial waves under the impact of viscous damping. As the most intriguing result, the theory predicts the formation of coherent spiral patterns visible in higher wave modes, which, to the best of our knowledge, have never been reported so far in the frameworks of non-parametric sloshing. The formation of similar rotating spiral patterns is known only from physically different Faraday experiments (Kiyashko et al. 1996). The reason for this is twofold. On the one hand, nearly all the sloshing literature has focused on the lowest natural sloshing frequency, in which no spirals can emerge. On the other hand, the majority of sloshing experiments concern one-phase free-surface wave systems, where damping rates are usually far too small to break the symmetry, and the eigenfrequencies are typically too high to excite stable and linear waves in the higher modes.

Our interfacial sloshing experiment can fill exactly this gap. By employing two-layer stratifications, we can easily excite stable higher-mode small-amplitude waves with moderate shaking frequencies. We therefore devote this article to the experimental verification of this hitherto unheeded sloshing phenomenon.

The paper is organised as follows. In $\S 2$, we explain the spiralisation phenomenon theoretically and classify different spiral regimes as functions of the key dimensionless groups through a newly introduced spiralisation parameter. In $\S 3$, we introduce our experimental set-up, which we have extended by the background-oriented schlieren measurement technique allowing for high-resolution measurements of interfacial wave patterns. Finally, we compare two different sets of experiments against the theoretical predictions in $\S 4$. 


\section{Spiral waves in cylindrical containers under orbital excitation}

(a)



(b)

Figure 1. (a) Schematic illustration of the orbitally excited cylindrical tank and (b) photograph of our experimental set-up. The cylinder of radius $R$ is filled with two immiscible liquids, $i=1,2$, of densities $\rho_{i}$, kinematic viscosities $v_{i}$ and layer heights $h_{i}$, which stably stratify due to gravity $g$ and form a distinct liquid-liquid interface of interfacial tension $\gamma$ at the position $z=\eta(r, \varphi)$. The orbital shaking table prescribes ideal circular motions of diameter $d_{s}$ and constant angular frequency $\Omega$ to the tank while maintaining a fixed orientation. A charge-coupled device (CCD) camera is mounted coaxially above the observation tank to allow direct image acquisition in the non-inertial frame of reference. A transparent random dot pattern placed just beneath the tank bottom, which is homogeneously illuminated from below by a light-emitting diode (LED) array, serves as the background.

\section{Theory}

In Horstmann et al. (2020), we derived an analytic solution describing the linear motion of gravity-capillary interfacial waves in upright circular cylinders undergoing a constant circular orbital motion. The defined theoretical framework is shown in figure 1 $(a)$. An ideal circular cylinder of radius $R$ contains two immiscible liquid layers (subscripts $i=$ $1,2)$ of heights $h_{i}$, kinematic viscosities $v_{i}$ and densities $\rho_{i}$. Under the constraint $\rho_{1}<\rho_{2}$, both liquids stably stratify due to gravity, $\boldsymbol{g}=-g \boldsymbol{e}_{z}$, and form a liquid-liquid interface at the position $z=\eta(r, \varphi)$. The origin of the cylindrical coordinate system $O(r, \varphi, z)$ is located in the centre of the interface $\eta$ and follows the tank motion. Interfacial tension $\gamma$ was incorporated to also describe the capillary wave regime. The interfacial contact line at the sidewall was assumed to move freely while maintaining a static contact angle of $90^{\circ}$ (no meniscus). This idealised boundary condition is only justified if the capillary length $\delta_{c}=\sqrt{\gamma /\left(\rho_{2}-\rho_{1}\right) g}$ is far smaller than the lateral dimensions of the vessel, which is safely satisfied by all liquid stratifications presented in this study, having a capillary length of approximately $2 \mathrm{~mm}$; see Bond numbers in table 1 . The entire vessel is oscillated horizontally with a constant angular frequency $\Omega$ along a circular trajectory of diameter $d_{S}$. 
Most concisely, this wave problem is governed by eight key dimensionless groups. In compliance with the free-surface sloshing theory by Reclari et al. (2014), we have chosen the following set of dimensionless numbers:

$$
\begin{gathered}
F r=\frac{d_{s} \Omega^{2}}{2 g}, \quad E=\frac{d_{s}}{2 R}, \quad R e_{i}=\frac{\Omega R^{2}}{\nu_{i}}, \quad H_{i}=\frac{h_{i}}{R}, \\
B o=\frac{\left(\rho_{2}-\rho_{1}\right) g R^{2}}{\gamma}, A=\frac{\rho_{2}-\rho_{1}}{\rho_{1}+\rho_{2}} .
\end{gathered}
$$

Here, $F r$ denotes the Froude number representing the forcing expressed by the ratio of inertial force and gravitational acceleration. The normalised shaking diameter $E$ can be interpreted as the eccentricity, while $R e_{i}$ are the fluid-dependent Reynolds numbers, here weighting the cylinder radius with the boundary layer thicknesses $\delta_{i} \sim \sqrt{v_{i} / \Omega}$. The importance of gravitational forces compared to interfacial tension forces is specified by the Bond number $B o$. Finally, $H_{i}$ denote the dimensionless aspect ratios and the Atwood number $A$ characterises the transition from interfacial waves (small $A$ ) to free-surface waves $(A \approx 1)$.

By introducing the dimensionless variables $\tilde{r}=r / R, \tilde{z}=z / R$ and $\tilde{t}=\Omega t$, we (Horstmann et al. 2020) expressed the linear solution for the dimensionless wave elevation $\eta(\tilde{r}, \varphi, \tilde{t}) / R$ in the following form:

$$
\begin{aligned}
\frac{\eta(\tilde{r}, \varphi, \tilde{t})}{R}= & \sum_{n=1}^{\infty} \frac{2 F r}{\left(1+\frac{\epsilon_{1 n}^{2}}{B o}\right)} \frac{\mathrm{J}_{1}\left(\epsilon_{1 n} \tilde{r}\right)}{\left(\epsilon_{1 n}^{2}-1\right) \mathrm{J}_{1}\left(\epsilon_{1 n}\right)} \\
& \times\left[\frac{\Gamma_{\omega_{1 n}}^{2}}{\frac{\left(\Gamma_{\omega_{1 n}}^{2}-1\right)^{2}}{\Lambda_{1 n}}+\Lambda_{1 n}} \sin (\tilde{t}-\varphi)+\frac{\Gamma_{\omega_{1 n}}^{2}}{\left.\left(\Gamma_{\omega_{1 n}}^{2}-1\right)+\frac{\Lambda_{1 n}^{2}}{\left(\Gamma_{\omega_{1 n}}^{2}-1\right)} \cos (\tilde{t}-\varphi)\right]}\right.
\end{aligned}
$$

Here $\Gamma_{\omega_{1 n}}:=\omega_{1 n} / \Omega$ are the dimensionless frequency ratios and $\Lambda_{1 n}:=2 \lambda_{1 n} / \Omega$ are the damping ratios, both of which are specified in Appendix A. The values $\lambda_{1 n}$ refer here to the two-layer viscous damping rates derived in Herreman et al. (2019, appendix D). Further, $\mathrm{J}_{1}$ denotes the first-order Bessel function of the first kind and the $\epsilon_{1 n}$ indicate the discrete wavenumbers, given as the $n$ roots of the first derivative of the first-order Bessel function, $\mathrm{J}_{1}^{\prime}\left(\epsilon_{1 n}\right)=0$ (Abramowitz \& Stegun 1972). Physically, the integers $n$ specify the number of antinodal cycles (crest-trough pairs) appearing in the wave excited with the frequency $\Omega=\omega_{1 n}$.

Perhaps the most intriguing feature of solution (2.2) is the description of phase lags developing between the wave motion and the orbital motion of the shaking table, which emerge in the presence of damping. The phase lag $\Delta \varphi$, explicitly given by

$$
\Delta \varphi(\tilde{r})=\arctan \left(\frac{\sum_{n=1}^{\infty} \frac{1}{\left(B o+\epsilon_{1 n}^{2}\right)} \frac{\mathrm{J}_{1}\left(\epsilon_{1 n} \tilde{r}\right)}{\left(\epsilon_{1 n}^{2}-1\right) \mathrm{J}_{1}\left(\epsilon_{1 n}\right)} \frac{\Lambda_{1 n} \Gamma_{\omega_{1 n}}^{2}}{\left(\Gamma_{\omega_{1 n}}^{2}-1\right)^{2}+\Lambda_{1 n}^{2}}}{\sum_{n=1}^{\infty} \frac{1}{\left(B o+\epsilon_{1 n}^{2}\right)} \frac{\mathrm{J}_{1}\left(\epsilon_{1 n} \tilde{r}\right)}{\left(\epsilon_{1 n}^{2}-1\right) \mathrm{J}_{1}\left(\epsilon_{1 n}\right)} \frac{\Gamma_{\omega_{1 n}}^{2}\left(\Gamma_{\omega_{1 n}}^{2}-1\right)}{\left(\Gamma_{\omega_{1 n}}^{2}-1\right)^{2}+\Lambda_{1 n}^{2}}}\right),
$$




\section{Spiral waves in cylindrical containers under orbital excitation}
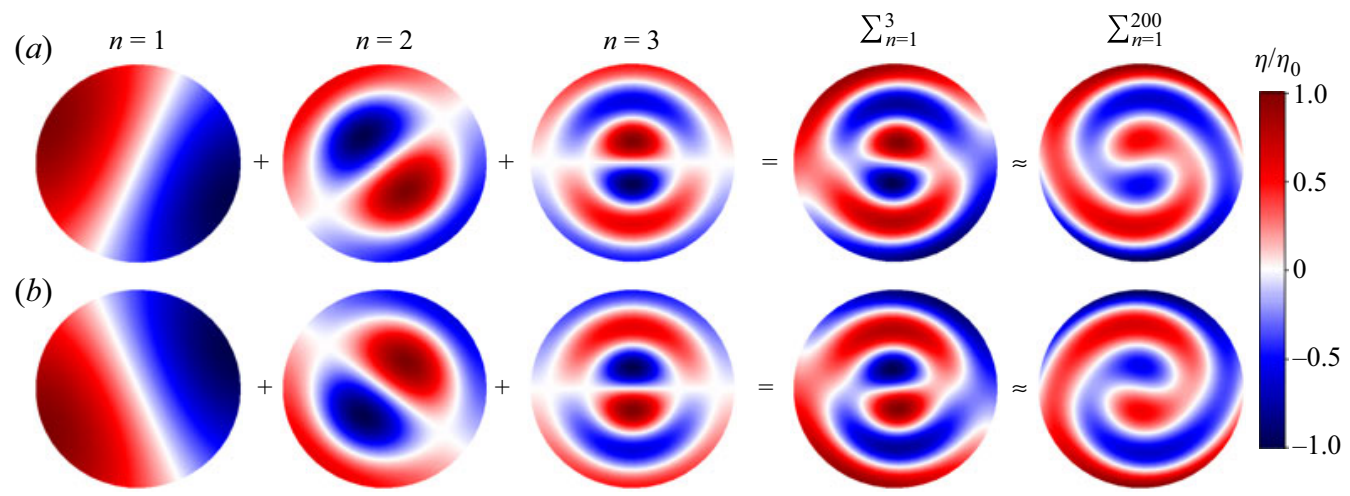

Figure 2. Spiral wave formation and origin of chirality highlighted by superimposing the first fundamental Fourier-Bessel modes for a clockwise-rotating orbit $(a)$ and the anticlockwise-rotating orbit $(b)$ of the third wave mode $\Omega=\omega_{13}$ for the coherent spiral case shown in figure 3 .

is an intricate function of the radial position $\tilde{r}$. As a consequence, the different antinodal cycles are subject to different phase shifts (with respect to the motion of the shaker), which are largely dictated by the damping ratios $\Lambda_{1 n}$. Exactly this effect is responsible for the spiral pattern formation since this position-dependent shifting introduces a relative twisting between the individual crest-trough pairs (antinodal cycles), letting the waves appear as one-armed spirals in superposition.

This mechanism, which differs fundamentally from the nonlinear spiral pattern formation occurring in reaction-diffusion systems and excitable media, deserves further explanation. In order to arrive at a more lucid understanding, we can graphically examine how the wave modes are composed of the fundamental Fourier-Bessel modes in the solution (2.2). Let us consider, by way of example, the third wave mode developing at the frequency $\Omega=\omega_{13} \Longleftrightarrow \Gamma_{\omega_{13}}=1$. To be formally exact, we would have to calculate this wave by summing up an infinite number of Fourier-Bessel modes $n$ in (2.2). However, only the first few summands contribute significantly to the wave mode, since the terms $\Gamma_{\omega_{1 n}}=1$, which appear in the denominators, are small (and therefore significant) only if $n$ closely matches, in this example, the third eigenfrequency. It is well known that the first $n$ fundamental Fourier-Bessel modes essentially define the wave structure of waves excited by the $n$th eigenfrequency. Higher terms only add small details.

All this is illustrated in figure 2, showing the first three Fourier-Bessel modes, its superposition as well as the converged solution of a typical $\Omega=\omega_{13}$ spiral wave pattern (compare to figure 2 in Horstmann et al. (2020)) resulting from clockwise (a) and anticlockwise (b) circular excitation. It can be seen how the ongoing twisting of the fundamental modes leads to spiralisation. In the classic inviscid solution, all Fourier-Bessel modes have the same phase or are phase shifted by exactly $180^{\circ}$ such that the axial symmetry of the single modes is preserved in the superimposed solution. The presence of damping, in contrast, inevitably breaks this symmetry. A remarkable consequence of this symmetry breaking is that spiral waves are characterised by a pronounced chirality defined by the rotational direction of the shaking table. Clockwise and anticlockwise spiral waves are, unlike the classical solutions, no longer indistinguishable. The mere presence of a dissipation source allows the wave to 'remember' the rotational sense of excitation. 


\section{G.M. Horstmann, S. Anders, D.H. Kelley and T. Weier}

Expanding on this, we would like to discuss the spiral wave terminology in some more detail. Unfortunately, there is no consistent definition of spiral waves in the literature. Most generally, a spiral is described by a continuous monotonic function $r=r(\varphi)$ in polar coordinates. Such a relationship is evident from the white-coloured nodal ridges visible in the converged solution in figure 2 , where the nodal radius is monotonically growing with the angle $\phi$. In terms of wave mechanics, however, we have to further distinguish between propagating and standing waves. Most spiral waves known, e.g. from reaction-diffusion systems, propagate either outwards from or inwards into the spiral centre (Vanag \& Epstein 2001). Such a radial propagation, also in contrast to vertically excited spiral Faraday waves (Kiyashko et al. 1996), is not present in our solutions. Equation (2.2) describes a kind of standing wave that azimuthally rotates as a whole like a solid body with the fixed frequency predetermined by the shaking table. Please note, however, that nonlinear streaming effects arising at the sidewall will always cause a radial mean mass transport in the bulk, which is largely associated with the interface elevation (Bouvard et al. 2017).

In Horstmann et al. (2020), we have illustrated this spiral formation for different fixed damping rates in order to stress this phenomenon in a simple way. However, the formation of spiral waves turned out to be far more complex when applying the individual damping ratios $\Lambda_{1 n}$ (A2), which can take considerably different values for the different wave modes $n$. Thereby, the wave crests and troughs do not necessarily merge into coherent spirals, but can become positionally separated and therefore incoherent. By way of examples, figure 3 shows different representative wave patterns that arise from solution (2.2). Essentially, we have identified four characteristic wave regimes: the well-known inviscid solutions, incoherent spirals with one or multiple discontinuities, continuous coherent spirals as well as overdamped waves, where the latter are defined by damping ratios larger than one $\Lambda_{\omega_{1 n}} \geq 1$, due to which they would rapidly decay within only one wave period after switching off the shaking table.

This result has led to the question of if and how we can classify these regimes as functions of the dimensionless groups. As a first step, we seek a suitable wave criterion quantifying the degree of spiralisation. A simple way is the pair-by-pair comparison of the crest or trough amplitudes in the $j=1,2, \ldots,(n-1)$ nodal cycles (visible in the inviscid solutions as white rings), located at the positions

$$
\tilde{r}_{n o d a l, j}=\frac{\kappa_{1 j}}{\left(\frac{\Gamma_{\omega_{1 n+1}}-1}{\Gamma_{\omega_{1 n+1}}-\Gamma_{\omega_{1 n}}}\right) \epsilon_{1 n}+\left(\frac{1-\Gamma_{\omega_{1 n}}}{\Gamma_{\omega_{1 n+1}}-\Gamma_{\omega_{1 n}}}\right) \epsilon_{1 n+1}}, \quad \text { for } \omega_{1 n} \leq \Omega<\omega_{1 n+1},
$$

with the $\pi / 2$ phase shifted inner amplitudes of the $j=1,2, \ldots, n$ antinodal cycles (crest-trough circles surrounding the white rings in the inviscid solution), located at the positions

$$
\tilde{r}_{\text {antinodal }, j}=\frac{\epsilon_{1 j}}{\left(\frac{\Gamma_{\omega_{1 n+1}}-1}{\Gamma_{\omega_{1 n+1}}-\Gamma_{\omega_{1 n}}}\right) \epsilon_{1 n}+\left(\frac{1-\Gamma_{\omega_{1 n}}}{\Gamma_{\omega_{1 n+1}}-\Gamma_{\omega_{1 n}}}\right) \epsilon_{1 n+1}}, \quad \text { for } \omega_{1 n} \leq \Omega<\omega_{1 n+1} .
$$

The numbers $\kappa_{1 j}$ are given as the $j$ zeros of the first-order Bessel function $\mathbf{J}_{1}\left(\kappa_{1 j}\right)=0$. In the case of resonant excitations $\left(\Omega=\omega_{1 n}\right)$, expressions (2.4) and (2.5) simplify to $\tilde{r}_{\text {nodal }, j}=\kappa_{1 j} / \epsilon_{1 n}$ and $\tilde{r}_{\text {antinodal }, j}=\epsilon_{1 j} / \epsilon_{1 n}$. 


\section{Spiral waves in cylindrical containers under orbital excitation}

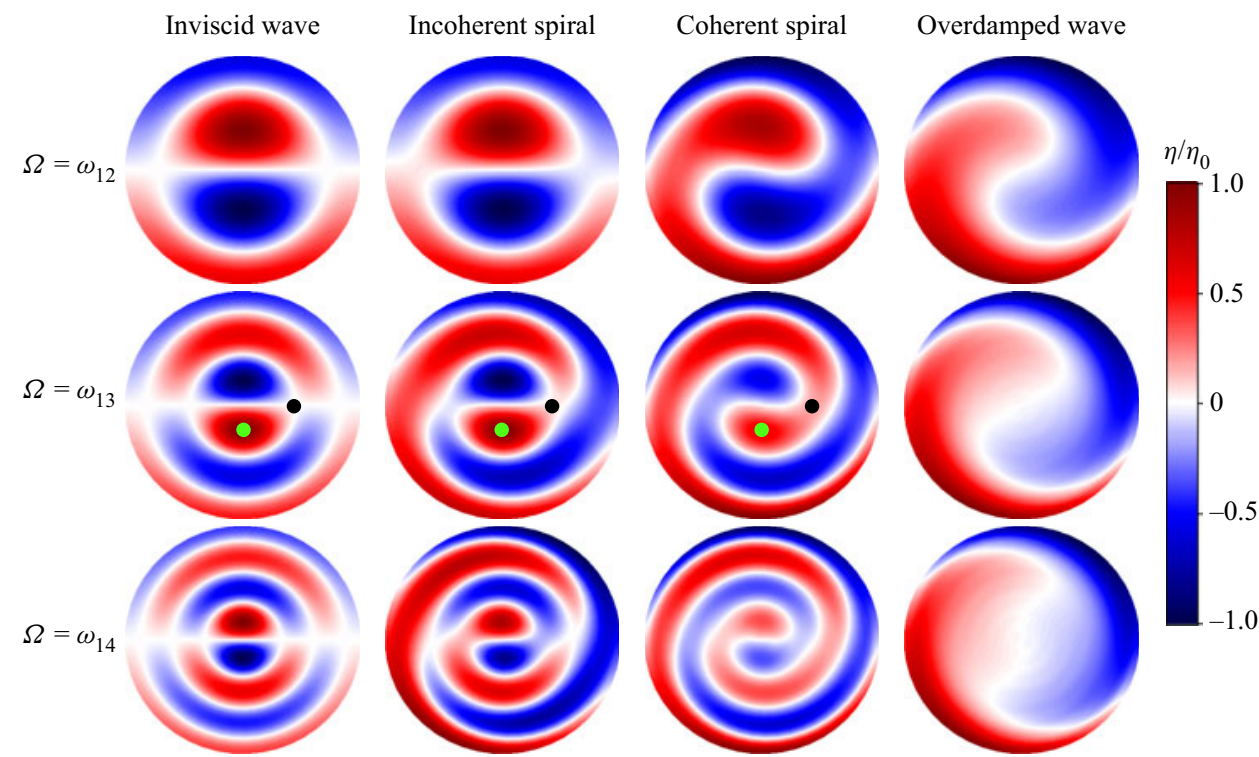

Figure 3. Different characteristic spiral wave regimes visualised for the first three spiralisable modes appearing at the eigenfrequencies $\Omega=\omega_{12}, \omega_{13}$ and $\omega_{14}$. The interface elevations $\eta(\tilde{r}, \varphi) / \eta_{0}$, here normalised by the maximum wave amplitude $\eta_{0}$, are calculated by applying the default parameters $E=1, B o=10^{4}$, $A=0.1, R e_{1}=R e_{2}=12.5 \times 10^{4} \sqrt{F r}, H_{1}=2-H_{2}$ and $H_{2}=0.8$ (incoherent spirals) in (2.2). The inviscid waves were created by taking the limits $R e_{1}=R e_{2} \gg 1$ and the overdamped waves by setting $R e_{1}=R e_{2}=$ $200 \sqrt{F r}$. Finally, the coherent spirals are obtained by $H_{2}=0.05$. In each case, $F r$ is chosen to correspond to the eigenfrequencies by setting $\Gamma_{\omega_{12}}, \Gamma_{\omega_{13}}$ and $\Gamma_{\omega_{14}}=1$ in accordance with (A1). Black and green circles mark crest locations on the first nodal and antinodal cycle, respectively (see text).

For a better clarification, the points $\tilde{r}_{\text {nodal }, 1}$ and $\tilde{r}_{\text {antinodal, } 1}$ are marked as black and green dots, respectively, in the $\Omega=\omega_{13}$ solutions in figure 3 . In the case of inviscid waves, the pair-to-pair amplitude ratios at $\tilde{r}_{\text {nodal, } j}$ compared to $\tilde{r}_{\text {antinodal, } j \text { are always zero, whereas }}$ they would approach one for perfectly homogeneous spirals. On this basis, we introduce the spiralisation parameter $\mathcal{S}$ as follows:

$$
\mathcal{S}:=\min \left(\left|\frac{\eta\left(\tilde{r}=\tilde{r}_{\text {nodal }, j}, \varphi=0\right)}{\eta\left(\tilde{r}=\tilde{r}_{\text {antinodal }, j}, \varphi=\pi / 2\right)}\right|\right), \quad j=1,2, \ldots,(n-1) .
$$

This parameter is suitable to analyse the spiralisation as a function of the dimensionless groups. Because spiral formation is a gradual process, there is no fixed threshold allowing one to distinguish sharply between coherent and incoherent spirals, but we observed numerically that spirals appear comparatively homogeneous and shaped like the coherent spirals presented in figure 3 for $\mathcal{S}>0.1 \pm 0.02$. Therefore, we propose $\mathcal{S} \gtrsim 0.1$ as a criterion to identify coherent spirals, whereas the regime $\mathcal{S} \lesssim 0.1$ comprises incoherent spiral waves as well as the inviscid solutions $(\mathcal{S}=0)$. Overdamped waves, in contrast, cannot be identified by means of the parameter since the inner nodal and antinodal cycles gradually disappear in this regime.

The numerical evaluation of $\mathcal{S}$ while varying all the dimensionless parameters $(2.1 a-f)$ revealed very complex spiralisation dynamics, with no obvious scaling dependences. Surprisingly, we found that the Reynolds numbers $R e_{i}$ are not the most critical parameters for achieving coherent spirals. The presence of damping is a necessary but not sufficient precondition. The Bond number $B o$ and the aspect ratios $H_{i}$ have a more significant 

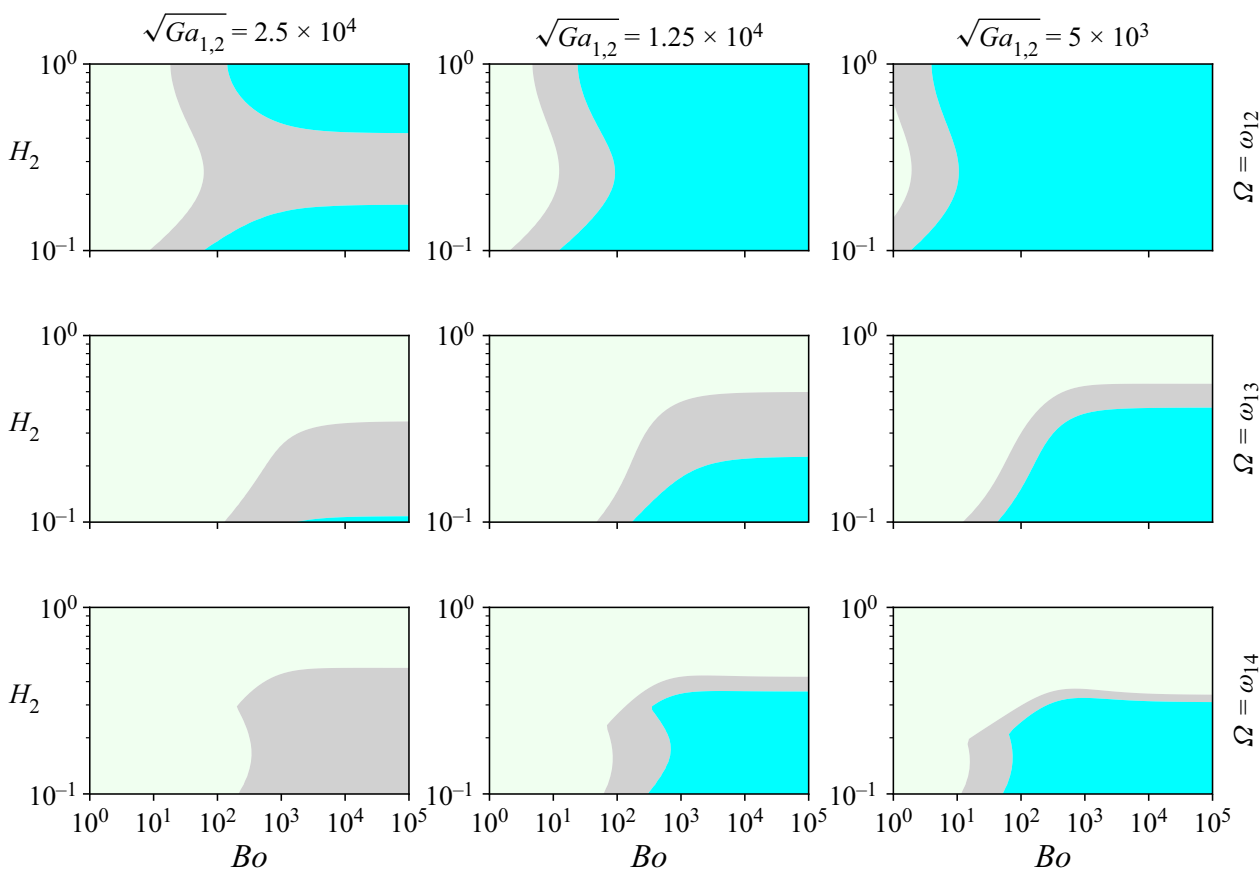

Incoherent spiral

Transitional regime

Coherent spiral

Figure 4. Spiral regimes visualised in $H_{2}-B o$ space for the wave modes $\Omega=\omega_{12}, \omega_{13}$ and $\omega_{14}$ and different Galilei numbers $\sqrt{G a_{i}}=R e_{i} / \sqrt{F r}=\sqrt{g R^{3} v^{2}}{ }_{i}$. The Froude numbers were chosen to meet the eigenfrequencies $\Gamma_{\omega_{1 n}}=1$ according to (A1), with $E=1, A=0.05$ and $H_{1}=2-H_{2}$. The coherent spiral regime is defined by $\mathcal{S}>0.12$, whereas $\mathcal{S}<0.08$ reflects incoherent spirals, including the classical inviscid solutions. In between, $0.08 \leq \mathcal{S} \leq 0.12$ highlights the gradual transition

influence, with the general tendency that large $B o$ (gravity wave limit) and shallow layers (either small $H_{1}$ or $H_{2}$ ) strongly favour coherent spirals. In contrast, $\mathcal{S}$ is independent of $E$ and depends only weakly on $A$ in the interfacial wave regime $A \lesssim 0.2$ considered here.

To provide a more quantitative understanding of the most important spiralisation characteristics, we study different parameter spaces around the accessible regimes of our experimental set-up introduced in $\S 3$. In the following, we set $E=1$ without loss of generality. Further, we choose $A=0.05$, a typical value for oil-water stratifications, assume symmetric viscosities $R e_{1}=R e_{2}$ for the sake of simplicity and fix the aspect ratio of the cylinder to $H_{1}+H_{2}=2$. Given these conditions, figure 4 shows the different spiral wave regimes in $\mathrm{H}_{2}-\mathrm{Bo}$ spaces for the first three spiralisable wave modes $\Omega=\omega_{12}, \omega_{13}$ and $\omega_{14}$ and different damping strengths, here expressed in terms of the frequency-independent Galilei numbers $\sqrt{G a_{i}}=R e_{i} / \sqrt{F r}=\sqrt{g R^{3} v_{i}^{2}}$. This reformulation was necessary to ensure comparability throughout the wave modes, which have very different eigenfrequencies. The three visualised spiral regimes represent different contour levels of $\mathcal{S}$, defined as follows: $\mathcal{S}<0.08$ represents classic waves and incoherent spirals; $0.08 \leq \mathcal{S} \leq 0.12$ marks the transitional regime towards coherent spirals; and $\mathcal{S}>0.12$ classifies clean coherent spirals. Although these boundaries were chosen somewhat arbitrarily on aesthetic grounds, figure 4 well reflects the overall spiralisation behaviour and reveals its complexity. The first presented mode $\Omega=\omega_{12}$ plays a small role of its own, 


\section{Spiral waves in cylindrical containers under orbital excitation}

as the two nodal cycles merge particularly easily. All other modes (also higher modes not shown here) exhibit very similar but not identical behaviour: coherent spirals are predicted to evolve only for sufficiently high Galilei numbers in the gravity wave and shallow water limits.

\section{Experiment}

In Horstmann et al. (2019), we established an acoustic measurement technique for the reconstruction of rotating interfacial waves, originally with the aim of measuring waves in opaque liquid-metal stratifications. However, the proper verification of the smaller-scale spiral wave patterns requires continuous measurements to high resolution. For this purpose, we advanced the experimental apparatus to apply the background-oriented schlieren (BOS) method (often also called synthetic schlieren). This technique can be applied to directly measure the instantaneous topography of interfacial slopes between transparent fluids with different refractive indices (Moisy, Rabaud \& Salsac 2009). The implementation is shown in figure $1(b)$. All experiments were conducted in a cylindrical vessel made from clean acrylic glass. The inner radius measures $R=50 \mathrm{~mm}$ and the total height of the inner volume was adjusted to $h_{1}+h_{2}=75 \mathrm{~mm}$. The entire observation cell is placed on a stand mounted on a $420 \mathrm{~mm} \times 420 \mathrm{~mm}$ shaking tray following ideal orbital motions excited by a modified Kuhner LS-X laboratory shaker. The shaker allows for a continuous adjustment of shaking diameters up to $d_{s}=70 \mathrm{~mm}$ and facilitates shaking frequencies $f=\Omega / 2 \pi$ in the range 20 r.p.m. $\leq f \leq 500$ r.p.m.

For the appropriate implementation of BOS, the experimental set-up from Horstmann et al. (2019) was extended by two components. For backlight illumination, we have installed a Luminitronix MiniMatrix array of $10 \times 10$ LEDs $(15 \mathrm{~cm} \times 15 \mathrm{~cm})$ below the vessel. To achieve a homogeneous diffusive backlight, we further mounted a $2 \mathrm{~mm}$ thick white sheet of polystyrene above the LED array. The emitted light passes vertically through the cylinder and is captured by an AVT Prosilica GT1660 monochrome camera. The camera was mounted coaxially $270 \mathrm{~mm}$ above the vessel, resulting in an imaging of the

fluid cell of $1000 \mathrm{px} \times 1000 \mathrm{px}$ with a resolution of $0.1 \mathrm{~mm} \mathrm{px}^{-1}$. In this arrangement the camera follows the orbital motion of the shaking table, which has the advantage that acquired images can be processed directly without the need to transform the frame of reference. Finally, a transparent background was installed just beneath the bottom of the fluid vessel. We generated a random dot pattern of $\sim 20$ dots $\mathrm{mm}^{-2}$ using the software PIVview that was printed onto a transparent plastic sheet.

The principle of BOS is based on the fact that local wave elevations are reflected as virtual displacements $\delta \boldsymbol{x}(r, \varphi)$ of the dot pattern with regard to a reference image obtained when the interface is flat. Light rays originating from points below the interface are refracted by a certain angle dependent on the local slope of the interface $\nabla \eta(r, \varphi)$ and the refractive indices of all involved phases. For better clarification of this principle, a supplementary movie is provided (available at https://doi.org/10.1017/jfm.2021.686) showing spiral wave motion becoming directly visible as caustics in the reference pattern.

Our theoretical model predicts that coherent spirals preferentially form if one of the fluid layers is shallow. Fortunately, restricting to shallow bottom layers $H_{2} \lesssim 0.25$ is also favourable for BOS measurements. It is shown by Moisy et al. (2009) that, if the reference pattern-interface distance is small and the camera-interface distance is large (paraxial approximation), the interfacial slope is linearly correlated with the displacement field,

$$
\nabla \eta(r, \varphi) \sim-\delta \boldsymbol{x}(r, \varphi)
$$


(a)

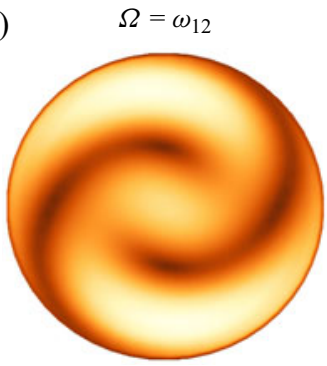

(b)

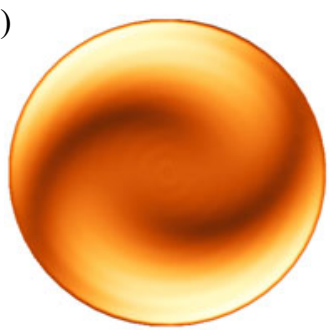

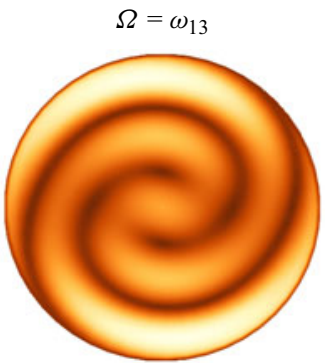

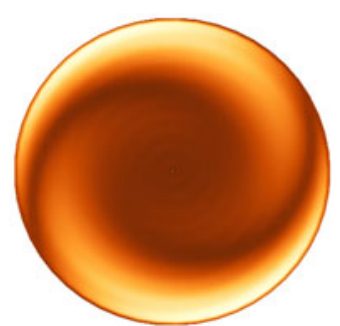

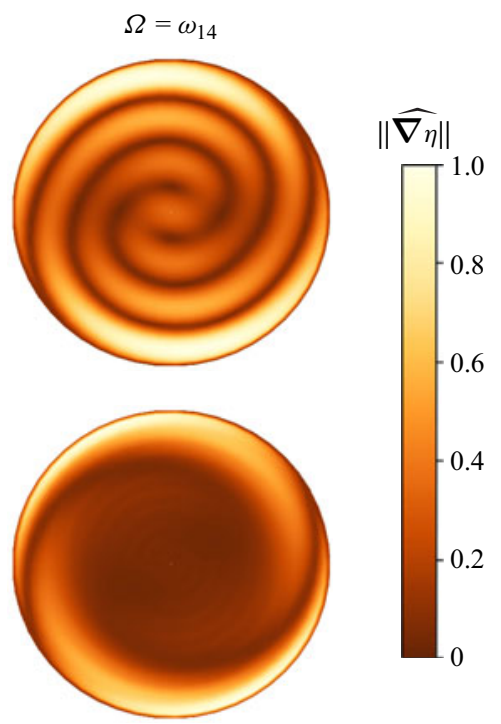

Figure 5. Normalised absolute values of the gradient fields $\|\widehat{\nabla \eta}\|$ due to (2.2) corresponding to the coherent spirals $(a)$ and overdamped solutions $(b)$ shown in figure 3 .

provided that wave amplitudes and slopes are small $\left\|\eta_{1 n}\right\| \ll R / \epsilon_{1 n}$, which was anyway a basic requirement for the linearisation of our model (Horstmann et al. 2020). In principle, it is possible to determine the proportionality analytically by reconstructing the ray paths and inverting (3.1). This would allow one to determine wave amplitudes quantitatively, as recently demonstrated by Simonini et al. (2021) for free-surface sloshing in cylindrical containers. However, in our case the gradient is analytically known from (2.2). Therefore, we can avoid all the uncertainties that would arise from the reconstruction of the multiple refracted light rays and the numerical inversion procedure of (3.1).

In order to verify the predicted spiral patterns with the highest accuracy, it is expedient to directly compare normalised gradient fields $\nabla \eta$ following from (2.2) with the experimental displacement fields $\delta \boldsymbol{x}$. This way, we compare the slope of the interface throughout space and time up to a single unknown scaling parameter, which is of no significance and can be eliminated through normalisation. Based on this concept, we determined $\delta \boldsymbol{x}$ from recorded schlieren patterns by correlating successive images using the software PIVview 3.6. All displacement fields presented below were phase-averaged and interpolated on a standardised $0.5 \mathrm{~mm} \times 0.5 \mathrm{~mm}$ Cartesian grid in order to increase the accuracy. This way, we could keep maximum measurement uncertainties in $\delta \boldsymbol{x}(r, \varphi)$ well below $\pm 0.1 \mathrm{~mm}$.

\section{Results}

On the basis of relation (3.1), we now seek to verify the predicted existence of linear spiral waves. First, we need to analyse the gradient fields as they follow from the solution (2.2). It is a well-known fact that the application of the gradient operator on two-dimensional spiral patterns doubles the number of spiral arms. Hence, the one-armed spiral patterns from figure 3 are expected to become visible as two-armed spirals in the schlieren images. For clarity, figure 5 shows the normalised magnitude of the gradient fields $\|\widehat{\nabla \eta}\|=\|\nabla \eta\| / \max (\|\nabla \eta\|)$ for the coherent and overdamped spirals from figure 3 . The coherent spirals are reflected by a continuously connected slope crest involving $(n+1)$ 


\begin{tabular}{|c|c|c|c|c|c|c|c|}
\hline Working liquid & $\begin{array}{c}f \\
\text { (r.p.m.) }\end{array}$ & $\begin{array}{c}d_{s} \\
(\mathrm{~cm})\end{array}$ & $\begin{array}{c}\rho_{i} \\
\left(\mathrm{~g} \mathrm{~cm}^{-3}\right)\end{array}$ & $\begin{array}{c}\gamma \\
\left(\mathrm{mN} \mathrm{cm}^{-1}\right)\end{array}$ & $\begin{array}{c}v_{i} \\
\left(\mathrm{~cm}^{2} \mathrm{~s}^{-1}\right)\end{array}$ & $\begin{array}{c}h_{i} \\
(\mathrm{~cm})\end{array}$ & $\begin{array}{c}R \\
(\mathrm{~cm})\end{array}$ \\
\hline $\begin{array}{l}\text { Paraffin oil (PP) } \\
\text { Silicone oil (AK 35) }\end{array}$ & $32-122$ & 2.5 & $\begin{array}{l}0.846 \\
0.955\end{array}$ & $<0.05$ & $\begin{array}{l}\approx 0.36 \\
\approx 0.35\end{array}$ & $\begin{array}{l}5.9 \\
1.6\end{array}$ & 5 \\
\hline $\begin{array}{l}\text { Paraffin oil (PP) } \\
\text { Water }\end{array}$ & $34-117$ & 0.9 & $\begin{array}{l}0.846 \\
0.998\end{array}$ & 0.072 & $\begin{array}{l}\approx 0.36 \\
\approx 0.01\end{array}$ & $\begin{array}{c}6.5 \\
1\end{array}$ & 5 \\
\hline Working liquid & $\mathrm{Fr}$ & E & A & Bo & $R e_{i}$ & $H_{i}$ & \\
\hline $\begin{array}{l}\text { Paraffin oil (PP) } \\
\text { Silicone oil (AK 35) }\end{array}$ & $0.014-0.208$ & 0.25 & 0.06 & $>534$ & $\begin{array}{l}\approx 233-887 \\
\approx 239-913\end{array}$ & $\begin{array}{l}1.18 \\
0.32\end{array}$ & \\
\hline $\begin{array}{l}\text { Paraffin oil (PP) } \\
\text { Water }\end{array}$ & $0.016-0.191$ & 0.09 & 0.082 & 518 & $\begin{aligned} & \approx 247-851 \\
\approx & 8901-30630\end{aligned}$ & $\begin{array}{l}1.3 \\
0.2\end{array}$ & \\
\hline
\end{tabular}

Table 1. Experimental parameters and corresponding dimensionless groups of the two conducted sets of experiments.

maxima along the vertical half-axis. In contrast, the inner slope maxima of the overdamped cases disappear progressively with increasing wave modes, leaving only two twisted outer slope maxima close to the sidewall.

The regime classification presented in $\S 2$ was used as the basis for planning two sets of experiments, one in which paraffin oil (paraffinum perliquidum, PP) was layered onto silicone oil (AK 35), and another in which we stratified PP on top of water. Both sets are specified in table 1 . From $\Gamma_{\omega_{1 n}}=1$, see (A2), we find the first theoretical eigenfrequencies at $\left(\omega_{11}, \omega_{12}, \omega_{13}, \omega_{14}\right)=(34.6,80,111,138)$ r.p.m. for PPlwater and at $\left(\omega_{11}, \omega_{12}, \omega_{13}, \omega_{14}\right)=(33.5,70,94,117)$ r.p.m. for PPIAK 35 such that the measured shaking frequency ranges 34-117 r.p.m. (PPlwater) and 32-122 r.p.m. (PPIAK 35) are expected to cover the first three and four eigenfrequencies, respectively. Generally, spirals are predicted to form for high Bond numbers $B o \gtrsim 10^{2}$, sufficiently high damping rates and shallow liquid layers $\mathrm{H}_{2} \lesssim 0.4$. All these requirements are met by both the PPIAK $35(0.3 \lesssim \mathcal{S} \lesssim 0.5$ in the range $32-122$ r.p.m. $)$ and PPlwater $(0.11 \lesssim \mathcal{S} \lesssim 0.15$ in the range 34-117 r.p.m.) systems, whereby the more viscous PPIAK 35 stratification $\left(\sqrt{G a_{1}} \approx\right.$ $\sqrt{G a_{2}} \approx 2000$ ) was expected to encompass the overdamped spiral regime as well, which is not reflected by the spiral parameter $\mathcal{S}$. In principle, it would be possible to reconstruct $\mathcal{S}$ from the BOS measurement in order to quantitatively assess the predicted regime boundaries. However, the complicated environment in two-layer experiments renders direct comparisons very difficult. The PP/water system suffers from a partially fixed contact line, whereas PPIAK 35 is subject to partial mixing. Both effects cause shifts in the eigenfrequencies (Horstmann et al. 2019) and can affect the damping rates. For this reason, here we restrict ourselves to proving the existence of the spiral wave regimes and their characteristic patterns; precise measurements of $\mathcal{S}$ are planned for future studies.

Figure 6 shows eight normalised displacement fields $\|\widehat{\delta \boldsymbol{x}}\|=\|\delta \boldsymbol{x}\| / \max (\|\delta \boldsymbol{x}\|)$ from both sets of experiments. In addition, we provide two supplementary movies, which encompass all measured displacement fields in sequence, thereby highlighting the progressively increasing spiral arm formation with increasing shaking frequencies. All measured displacement fields accurately reflect the theoretically predicted double-armed spiral patterns and therefore confirm their existence. As expected, spirals are not visible for 
(a)

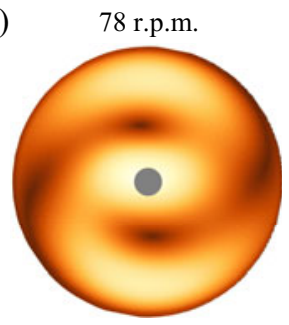

(b)

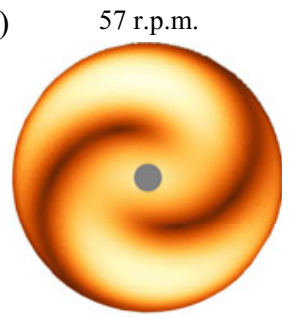



72 r.p.m.

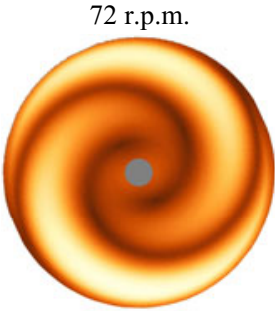

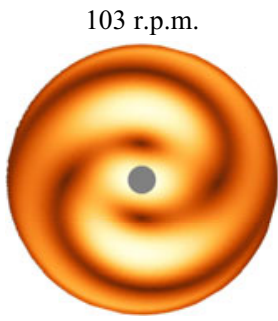

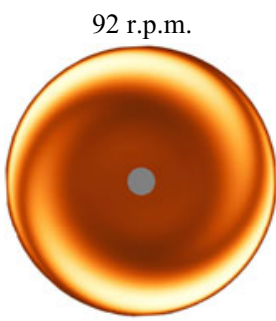

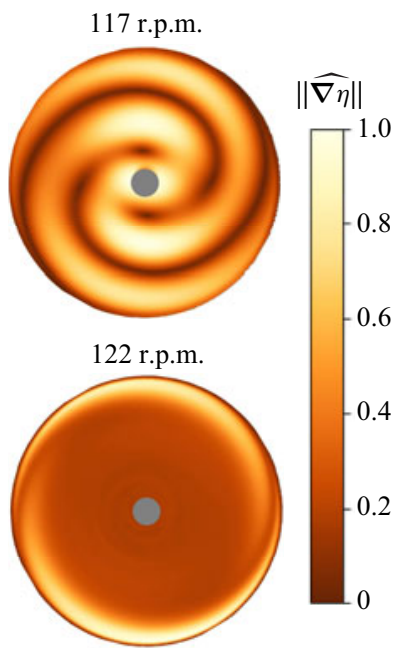

Figure 6. Normalised displacement fields $\|\widehat{\delta \boldsymbol{x}}\|$ for different chosen excitation frequencies in the range $\omega_{11} \lesssim f<\omega_{14}$ measured in PPlwater $(a)$ and PPIAK $35(b)$.

the lowest frequencies, since the first mode only involves one nodal cycle. From the second eigenfrequency on, the PPIwater system forms coherent spirals. The PPIAK 35 system encompasses further the transition into the overdamped regime. With frequencies above $f \gtrsim 75$ r.p.m. the inner structures start to vanish until the predicted overdamped patterns arise in the third and fourth mode. This is remarkable insofar as these cases already fall outside the low damping limit $R e_{i} \gg 1$ of the irrotational theory.

\section{Conclusion}

Building on our recent formulation of damped interfacial waves in orbitally shaken upright circular cylinders (Horstmann et al. 2020), we explain the emergence of novel spiral wave patterns resulting from the linear superposition of phase-shifted eigenmodes. We develop an analytic criterion quantifying the degree of spiralisation that allows us to classify the transitions from the classical inviscid wave modes to coherent spiral waves. The criterion reveals that spiral waves preferentially form in shallow layers and for high Bond numbers under the effect of sufficient damping. So as to be able to verify the existence of the predicted spiral wave regimes experimentally, we supplemented our existing multilayer orbital sloshing experiment (Horstmann et al. 2019) by the BOS measurement technique. This technique permits, in an easy way, submillimetre-resolution measurements of interfacial gradient fields, which are directly reflected in local displacements of a random dot pattern installed below the observation vessel. We performed two sets of experiments. By stratifying paraffin oil above water, the formation of coherent spirals could be confirmed for the second and third wave mode. Combining paraffin oil with a more viscous silicone oil instead, we further observed the formation of overdamped spirals in the third and fourth mode. These results confirm the existence of this novel class of linear spirals and substantiate the applicability of potential flow approaches also for damped wave mechanics. The spiralisation discussed here is a particular response to orbital excitation. For future studies, it would therefore be interesting to explore the linear and nonlinear wave patterns evolving under arbitrary (harmonic) excitations due to the same symmetry breaking that is a universal characteristic of higher-mode sloshing. 


\section{Spiral waves in cylindrical containers under orbital excitation}

Supplementary movies. Supplementary movies are available at https://doi.org/10.1017/jfm.2021.686.

Funding. This work was partially supported by a grant from the National Science Foundation (CBET1552182).

Declaration of interests. The authors report no conflict of interest.

\section{Author ORCIDs.}

(1) G.M. Horstmann https://orcid.org/0000-0001-9892-9309;

(1) D.H. Kelley https://orcid.org/0000-0001-9658-2954.

\section{Appendix A. Dimensionless frequencies and damping ratios}

Eigenfrequencies:

$$
\Gamma_{\omega_{1 n}}=\frac{\omega_{1 n}}{\Omega}=\left\{\frac{E}{F r} \frac{2 A \epsilon_{1 n}\left(1+\frac{\epsilon_{1 n}^{2}}{B o}\right)}{\left[(1-A) \operatorname{coth}\left(\epsilon_{1 n} H_{1}\right)+(1+A) \operatorname{coth}\left(\epsilon_{1 n} H_{2}\right)\right]}\right\}^{1 / 2} .
$$

Damping ratios for interfacial waves:

$$
\begin{aligned}
& \Lambda_{1 n}=\frac{2 \lambda_{1 n}^{2 \mathrm{~L}}}{\Omega} \\
& =\sum_{i=1,2} \sqrt{\frac{1}{2 R e_{i}}}\left[\frac{(i-1)(1+A)+(2-i)(1-A)}{\left[(1-A) \operatorname{coth}\left(\epsilon_{1 n} H_{1}\right)+(1+A) \operatorname{coth}\left(\epsilon_{1 n} H_{2}\right)\right]}\right] \\
& \times\left[\left(\epsilon_{1 n}-H_{i}\right) \sinh ^{-2}\left(\epsilon_{1 n} H_{i}\right)+\left(\frac{\epsilon_{1 n}^{2}+1}{\epsilon_{1 n}^{2}-1}\right) \operatorname{coth}\left(\epsilon_{1 n} H_{i}\right)\right] \\
& +\frac{\epsilon_{1 n}}{\left(\frac{\sqrt{2 R e_{1}}}{(1-A)}+\frac{\sqrt{2 R e_{2}}}{(1+A)}\right)}\left[\frac{\left[\operatorname{coth}\left(\epsilon_{1 n} H_{1}\right)+\operatorname{coth}\left(\epsilon_{1 n} H_{2}\right)\right]^{2}}{(1-A) \operatorname{coth}\left(\epsilon_{1 n} H_{1}\right)+(1+A) \operatorname{coth}\left(\epsilon_{1 n} H_{2}\right)}\right] \\
& +4 \epsilon_{1 n}^{2}\left[\frac{\frac{(1+A)}{R e_{2}}-\frac{(1-A)}{R e_{1}}}{\frac{(1-A)}{\sqrt{R e_{1}}}+\frac{(1+A)}{\sqrt{R e_{2}}}}\right]\left[\frac{\frac{(1+A)}{\sqrt{R e_{2}}} \operatorname{coth}\left(\epsilon_{1 n} H_{2}\right)-\frac{(1-A)}{\sqrt{R e_{1}}} \operatorname{coth}\left(\epsilon_{1 n} H_{1}\right)}{(1-A) \operatorname{coth}\left(\epsilon_{1 n} H_{1}\right)+(1+A) \operatorname{coth}\left(\epsilon_{1 n} H_{2}\right)}\right] .
\end{aligned}
$$

\section{REFERENCES}

Abramowitz, M. \& Stegun, I.A. 1972 Handbook of Mathematical Functions, 10th edn. Applied Mathematics Series, vol. 55. National Bureau of Standards.

Alpresa, P., Sherwin, S., Weinberg, P. \& VAn Reeuwijk, M. 2018 Orbitally shaken shallow fluid layers. II. An improved wall shear stress model. Phys. Fluids 30 (3), 032108.

BofFin, H.M.J. 2001 Spiral waves in accretion discs - theory. In Astrotomography: Indirect Imaging Methods in Observational Astronomy (ed. H.M.J. Boffin, J. Cuypers \& D. Steeghs), pp. 69-87. Springer.

Bouvard, J., Herreman, W. \& Moisy, F. 2017 Mean mass transport in an orbitally shaken cylindrical container. Phys. Rev. Fluids 2 (8), 084801.

Faltinsen, O.M. \& TimoKha, A.N. 2009 Sloshing. Cambridge University Press. 


\section{G.M. Horstmann, S. Anders, D.H. Kelley and T. Weier}

Faltinsen, O.M. \& Timoкha, A.N. 2019 An inviscid analysis of the Prandtl azimuthal mass transport during swirl-type sloshing. J. Fluid Mech. 865, 884-903.

GraY, R.A. \& JALIFE, J. 1996 Spiral waves and the heart. Intl J. Bifurcation Chaos 06 (03), 415-435.

Herreman, W., Nore, C., Guermond, J.-L., Cappanera, L., Weber, N. \& Horstmann, G.M. 2019 Perturbation theory for metal pad roll instability in cylindrical reduction cells. J. Fluid Mech. 878, 598-646.

Horstmann, G.M., Herreman, W. \& Weier, T. 2020 Linear damped interfacial wave theory for an orbitally shaken upright circular cylinder. J. Fluid Mech. 891, A22.

Horstmann, G.M., Weber, N. \& Weier, T. 2018 Coupling and stability of interfacial waves in liquid metal batteries. J. Fluid Mech. 845, 1-35.

Horstmann, G.M., Wylega, M. \& Weier, T. 2019 Measurement of interfacial wave dynamics in orbitally shaken cylindrical containers using ultrasound pulse-echo techniques. Exp. Fluids 60 (4), 56.

KiYashio, S.V., Korzinov, L.N., Rabinovich, M.I. \& Tsimring, L.S. 1996 Rotating spirals in a Faraday experiment. Phys. Rev. E 54 (5), 5037-5040.

KLÖCKNER, W. \& BÜCHS, J. 2012 Advances in shaking technologies. Trends Biotechnol. 30 (6), 307-314.

Moisy, F., Rabaud, M. \& SAlsac, K. 2009 A synthetic Schlieren method for the measurement of the topography of a liquid interface. Exp. Fluids 46 (6), 1021.

NOLAN, D.S. \& ZHANG, J.A. 2017 Spiral gravity waves radiating from tropical cyclones. Geophys. Res. Lett. 44 (8), 3924-3931.

Politis, G. \& Priede, J. 2021 Fractality of metal pad instability threshold in rectangular cells. J. Fluid Mech. 915, A101.

PRAndtL, L. 1949 Erzeugung von Zirkulationen beim Schütteln von Gefäßen. Z. Angew. Math. Mech. 29 (1-2), 8-9.

Reclari, M., Dreyer, M., Tissot, S., Obreschkow, D., Wurm, F.M. \& Farhat, M. 2014 Surface wave dynamics in orbital shaken cylindrical containers. Phys. Fluids 26 (5), 052104.

Simonini, A., Fontanarosa, D., De Giorgi, M.G. \& Vetrano, M.R. 2021 Mode characterization and damping measurement of liquid sloshing in cylindrical containers by means of Reference Image Topography. Exp. Therm. Fluid Sci. 120, 110232.

VANAG, V.K. \& EPSTEIN, I.R. 2001 Inwardly rotating spiral waves in a reaction-diffusion system. Science 294 (5543), 835-837.

Weber, N., Beckstein, P., Herreman, W., Horstmann, G.M., Nore, C., Stefani, F. \& Weier, T. 2017 Sloshing instability and electrolyte layer rupture in liquid metal batteries. Phys. Fluids 29 (5), 054101

Weheliye, W.H., Cagney, N., Rodriguez, G., Micheletti, M. \& Ducci, A. 2018 Mode decomposition and Lagrangian structures of the flow dynamics in orbitally shaken bioreactors. Phys. Fluids $30(3), 033603$. 